\title{
1 Comprehensive analysis of mutational signatures in pediatric cancers
}

2

3 Venu Thatikonda ${ }^{1,2,3}$, S. M. Ashiqul Islam ${ }^{4}$, Barbara C. Jones ${ }^{1,3,5,6}$, Susanne N. Gröbner ${ }^{1,2,3}$,

4 Gregor Warsow ${ }^{7}$, Barbara Hutter ${ }^{3,8,9}$, Daniel Huebschmann ${ }^{3,5,8,10}$, Stefan Fröhling ${ }^{3,8,11}$, Mirjam

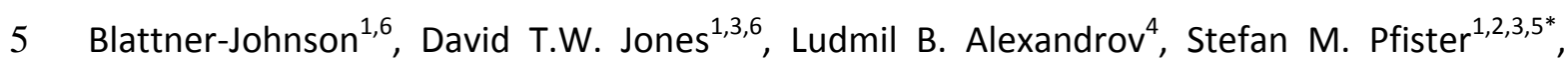

6 Natalie Jäger ${ }^{1,2,3^{*}}$

7

8 1. Hopp Children's Cancer Center Heidelberg (KiTZ), Heidelberg, Germany

9 2. Division of Pediatric Neurooncology, German Cancer Research Center (DKFZ), Heidelberg, Germany

10 3. German Cancer Consortium (DKTK), 69120 Heidelberg, Germany

4. Department of Cellular and Molecular Medicine and Department of Bioengineering, Moores Cancer Center, UC San Diego, La Jolla, 92093, California, USA

5. Department of Pediatric Oncology, Hematology and Immunology, Heidelberg University Hospital, Heidelberg, Germany

6. Pediatric Glioma Research Group, German Cancer Research Center (DKFZ), Heidelberg, Germany

7. Omics IT and Data Management Core Facility (W610), DKFZ, Heidelberg, Germany

8. NCT Molecular Diagnostics Program, NCT Heidelberg and DKFZ, 69120 Heidelberg, Germany

9. Division of Applied Bioinformatics, DKFZ, Heidelberg, Germany

10. Pattern Recognition and Digital Medicine, Heidelberg Institute for Stem cell Technology and Experimental Medicine (HI-STEM), Heidelberg, Germany

11. Division of Translational Medical Oncology, NCT Heidelberg and DKFZ, 69120 Heidelberg, Germany

* corresponding authors 


\section{Abstract}

27

Analysis of mutational signatures can reveal the underlying molecular mechanisms of the processes that have imprinted the somatic mutations found in a cancer genome. Here, we present a pan-cancer mutational signatures analysis of single base substitutions (SBS) and small insertion and deletions (ID) in pediatric cancers encompassing 537 whole genome sequenced tumors from 20 molecularly defined cancer subtypes. We identified only a small number of mutational signatures active in pediatric cancers when compared to the previously analyzed adult cancers. Further, we report a significant difference in the proportion of pediatric tumors which show homologous recombination repair defect signature SBS3 compared to prior analyses. Correlating genomic alterations with signature activities, we identified an association of TP53 mutation status with substitution signatures SBS2, SBS8, SBS13 and indel signatures ID2 and ID9, as well as chromothripsis associated with SBS8, SBS40 and ID9. This analysis provides a systematic overview of COSMIC v.3 SBS and ID mutational signatures active across pediatric cancers, which is highly relevant for understanding tumor biology as well as enabling future research in defining biomarkers of treatment response.

\section{Main}

Childhood cancers have a lower incidence rate when compared to the overall incidence of adult cancers. Nevertheless, cancer remains one of the leading causes of death by disease amongst children ${ }^{1}$. Growing research evidence suggests that childhood cancers are significantly different in terms of molecular features and therapy response when compared to their adult counterparts. Most prominently, recent pan-cancer analyses revealed that pediatric cancers show a significantly lower mutation burden in contrast to common adult cancers ${ }^{2,3}$. However, the knowledge of underlying mutational processes that contribute to the somatic mutation burden and tumor development in pediatric cancer is still limited.

Somatic mutations including single base substitutions, small insertions and deletions (indels), copy number changes, and other genomic rearrangements can be caused solely by endogenous processes (e.g., defects in DNA repair, errors in DNA replication, damage due to reactive oxygen species, etc.), or by the additional influence of exogenous causes such as exposure to ultra-violet light, tobacco smoking, and numerous others ${ }^{4}$. Previous pan-cancer 
59 analyses mainly focused on adult cancer types, employed mathematical models based on 60 nonnegative matrix factorization (NMF) to identify patterns of somatic mutations, termed 61 mutational signatures and utilized these patterns to infer underlying mutational processes ${ }^{5}$. 62 Since then, mutational signatures have been used to understand tumor development, to 63 identify gene alterations associated with mutational processes and importantly, as biomarkers 64 for predicting treatment response ${ }^{6,7,8,9,10,11,12}$. A total of 45 single base substitution signatures 65 (SBS) and 17 small insertion/deletion (ID) signatures were identified in a recent analysis as 66 part of the pan-cancer analysis of whole genomes (PCAWG) consortium ${ }^{13,14}$, updating and 67 expanding the previous set of reference mutational signatures (i.e., mutational signatures in 68 COSMIC v.2).

70 Previous studies of individual pediatric tumor types and pan-cancer approaches analyzed mutational signatures as part of molecular tumor landscape analyses using the COSMIC v.2 72 reference signatures ${ }^{15,16,17,18,19,20,23}$. Here, we carried out an extensive analysis encompassing 73 signatures of single base substitution and, for the first time, signatures of small 74 insertion/deletions in 537 whole genome sequenced pediatric tumor-normal pairs. These were 75 subsequently compared with COSMIC v. $3^{14}$ signatures to identify overlap with the latest set 76 of known mutational signatures. 


\section{Results}

\section{Somatic mutation frequencies across twenty pediatric cancer types}

A dataset of 537 whole genome sequenced tumor-normal pairs (the PedPanCan (PPC-WGS) cohort, https://www.kitz-heidelberg.de/en/research/datacommons/pedpancan/) was compiled from a previously published study ${ }^{2}$, spanning 20 molecularly defined entities of childhood cancer (Fig. 1a). Single base substitutions (SBS) and small insertions and deletions (IDs) were identified using an updated in-house mutation calling pipeline as compared with the previous analysis, with minor variations mostly in the calling strategy of indels (Supp. Fig. 1a). In total, across the cohort we identified 2,712,521 SBS and 270,928 IDs. The number of SBS mutations per megabase (median: 0.24; range: 0.0035 - 645.70) and IDs (median: 0.033; range: 0.00142- 46.64) was highly variable both across individual tumors as well as across different cancer types (Fig. 1a, Supp. Table 1). The lowest overall somatic mutation burden was observed in pilocytic astrocytoma (median: 0.0429 mutations per megabase) and the highest in osteosarcoma (median: 1.17). Although the indel mutation burden per tumor was low, numbers of SBS and IDs were significantly correlated across tumors (Fig. 1b). As previously described in different childhood and adulthood cancer types, the mutation burden of both SBS and IDs is also clearly correlated with age in this pediatric cohort (Supp. Fig. 1b). A small number $(n=3)$ of tumors classified as high-grade glioma with germline alterations in DNA mismatch repair genes (MSH6, PMS2) showed a hyper-mutator phenotype ${ }^{2}$, with 78.98 SBS and 2.63 ID mutations/Mb, respectively (Fig. 1b).

\section{SBS and ID signature activities}

To extract mutational signatures active in this pediatric pan-cancer cohort, we generated 96context SBS and 83-context ID mutational catalogues (Fig. 2a) and utilized an approach based on nonnegative matrix factorization, as previously described ${ }^{5,21,14}$. Due to the very low number of double base substitutions per pediatric tumor (data not shown), we only extracted signatures from SBS and IDs.

In total, 27 SBS signatures were extracted that matched the COSMIC v.3 SBS signatures with a minimum cosine similarity of 0.9 (Fig. 2b, Supp. Fig. 2a). Amongst these, SBS1 and SBS5 were present in $97.5 \%$ and $96.1 \%$ of samples across the cohort, respectively (Fig. 2b, Supp. Table 2, Supp. Table 3). As described in adult cancers and a small fraction of pediatric brain tumors, the clock-like nature of SBS1 and SBS5 was also observed in this cohort, with a 
113 significant correlation of signature activity with age at diagnosis ${ }^{7}$ (Supp. Fig. 3a). An 114 additional signature with unknown etiology, namely SBS40, is similar to SBS5 (cosine 115 similarity $=0.83)^{14}$. SBS40 was found to be active in five pediatric cancer types and $20 \%$ of 116 tumors across the cohort (Fig. 2b, Supp. Table 3) and was also correlated with age at 117 diagnosis (Supp. Fig. 3a), suggesting it may be an additional clock-like signature.

119 SBS2 and SBS13 were reported to be mainly due to the activity of APOBEC enzymes ${ }^{14}$ and 120 in this cohort they were found to be present only in adrenocortical carcinoma (ACC), high121 grade glioma and osteosarcoma (Fig. 2b, Supp. Table 2). In a cross-cohort comparison, 122 mutations attributed to SBS2 and SBS13 were significantly higher in TP53 germline mutated 123 tumors compared to tumors with somatic or no TP53 mutations (Supp. Fig. 3b). This 124 observation is in line with previous studies which identified a link between p53 loss and elevated $A P O B E C 3 B$ expression ${ }^{22,23}$. However, not all TP53 germline mutated tumors in this cohort showed SBS2 or SBS13 activity, including the TP53-defined subtype of SHHmedulloblastoma (Supp. Table 2 \& Table 13).

Ultraviolet light (UV) exposure signatures SBS7a and SBS7b ${ }^{14}$ were identified in tumors of hypodiploid B-cell acute lymphoblastic leukemia (B-ALL-HYPO) (Fig. 2b), but not in nonaneuploid tumors. UV light affected tumors show an enrichment of dipyrimidine substitution mutations (CC>TT). Consistently, SBS7a and SBS7b positive tumors show a significantly higher number of CC>TT doublet base substitutions when compared to SBS7a and SBS7b negative tumors in the B-ALL-HYPO subtype (Supp. Fig. 3c). Although multiple independent studies have recently identified signatures apparently linked with UV light exposure in pediatric B-ALL ${ }^{3,45}$, the exact mechanism how it contributes to leukemogenesis, and whether UV light is really the cause or rather the signature is mimicked by another process, remains to be elucidated. In 1.5\% of tumors (8/537; ETMR and Group4 medulloblastoma) we identified signature SBS4 (Fig. 2b, Supp. Table 2), which is proposed to be the result of DNA damage caused by tobacco smoking ${ }^{14}$. To the best of our knowledge, these tumors were derived from untreated, primary pediatric tumors and we hypothesize that the $\mathrm{C}>\mathrm{A}$ mutation pattern consistent with SBS4 may potentially be due to a different exogenous mutational process generating a similar signature of mutations as tobacco smoking. No common germline or 
146 Other relatively frequent signatures across cancer types included SBS8 and SBS18 (Fig. 2b).

147 Signature 8 from COSMIC v.2 was proposed to be due to homologous recombination repair

148 pathway gene mutations ${ }^{2}$. We did not identify any such mutations in SBS8 positive pediatric

149 tumors in this analysis. However, the number of mutations attributed to SBS8 was

150 significantly higher in tumors with germline or somatic TP53 mutations compared to wildtype

151 tumors (Supp. Fig. 3d). Recent evidence suggests that the SBS8 signature is due to the DNA

152 damage caused by late replication errors ${ }^{24}$. SBS18 is the result of DNA damage caused by

153 reactive oxygen species ${ }^{14}$ and is observed in 12 out of 20 cancer types analyzed in this cohort

154 (Fig. 2b). Amongst these 12 cancer types, the highest fraction of tumors with SBS18 signature

155 activity were observed in neuroblastoma (85\%, NB) and rhabdomyosarcoma (83\%, RMS)

156 (Supp. Table 4). Further, we found high activity of SBS18 in MYCN-amplified tumors in a

157 cross-cohort analysis (Supp. Fig. 3h). However, in a per-cancer-type analysis of NB, RMS

158 and Group3 medulloblastoma, tumors in which MYCN amplification is common, we did not

159 identify a significant SBS18 association with MYCN status.

160

161 Mutations attributed to signature SBS9 are due to DNA damage caused by polymerase eta

162 activity and were observed only in Burkitt’s lymphoma (BL) in this cohort. As previously

163 described, SBS9 activity is found in BL with immunoglobulin gene hypermutations ${ }^{36}$ (Supp.

164 Fig. 3e). Signature 10 from COSMIC v.2 has been split into SBS10a and SBS10b in the

165 COSMIC v.3 signatures ${ }^{14}$. Mutations from high-grade glioma hyper-mutator tumors were

166 attributed to both SBS10 signatures, as well as SBS14 and SBS15 (Fig. 2b). In Group4

167 medulloblastoma, we identified a novel SBS signature (termed Group4MB-SBS96D) with

168 elevated T>A mutations in the context of CTT/TTT (Supp. Fig. 3f, Supp. Table 5). This

169 signature contributed a large fraction of somatic point mutations to two tumors (3,623 in

170 ICGC_MB174 and 1,458 in ICGC_MB175, respectively). However, we did not identify any

171 molecular features common and specific to these tumors. Further investigation on the

172 processing of tumor material revealed that the surgery for these two patients was performed

173 on the same day in the same hospital, and therefore we suspect that the Group4MB-SBS96D

174 signature is an additional artefact signature.

175

176 In addition, we identified SBS21 in high-grade glioma, which is the result of defective DNA

177 mismatch repair, SBS36 in ETMR and Group4 medulloblastoma, which is the result of

178 defective base excision repair, and SBS44 in Group4 medulloblastoma, which is also caused

179 by defective DNA mismatch repair. For these signatures, we have not identified any 
180

181

182

183

184

185

186

187

188

189

190

191

192

193

194

195

196

197

198

199

200

201

202

203

204

205

206

207

208

209

210

211

212

213

associated consistent genetic alteration enriched across tumors. However, individual tumors harbor non-synonymous somatic mutations in different genes involved in DNA mismatch repair and nucleotide excision repair pathways (e.g. TP53, POLD1, SMG1, TP73, SWI5; Supp. Table 6). The possibility that tumors with SBS21, SBS36 and SBS44 activity might have epigenetic alterations affecting known genes in repair pathways and/or mutations in genes that play a role in repair pathways, but for which their function is not yet well characterized cannot be excluded.

Next, we sought to identify any associations between SBS signature activity, chromothripsis and genomic instability. In a cross-cohort analysis, mutations attributed to SBS8 and SBS40 were significantly higher in chromothriptic tumors (Supp. Fig. 3g and Supp. Table 12 for an overview of samples with chromothripsis). Individual cancer type analysis revealed that SBS40 activity in SHH-subtype medulloblastoma was significantly different depending on the presence of chromothripsis in the tumor genome (Supp. Fig. 3g). Genomic instability, quantified here as the total number of structural variants (deletions, duplications, inversions and translocations) identified in a tumor, is highly correlated with SBS2, SBS13 and SBS40 in a cross-cohort analysis (Supp. Fig. 3i). A separate per-cancer type analysis revealed a high correlation of genomic instability and SBS40 activity in SHH and Group4 medulloblastoma, as well as in neuroblastoma (Supp. Fig. 3i). These observations may indicate that mechanisms involved in genomic rearrangements contribute to mutational processes underlying APOBEC signatures and SBS40.

A total of nine small insertion/deletion (ID) signatures were identified from our PedPanCan cohort that matched with the COSMIC v.3 ID signatures (Fig. 2b, Supp. Table 7). As also observed in the PCAWG mutational signature analysis, ID1, ID2, ID5 and ID9 were active across tumors of multiple cancer types (Fig. 2b, Supp. Fig. 2b) ${ }^{14}$. ID1 and ID2, which are the result of DNA damage induced by replication slippage, were present in $~ 95 \%$ and $\sim 61 \%$ of the tumors in this cohort, respectively (the most prevalent ID signatures in this cohort). Although ID5 and ID9 were present in multiple cancer types, mutations were attributed to these signatures in only a small percentage of tumors (Supp. Table 8, Supp. Table 9). ID8, a signature caused by the potential damage induced by DNA double-strand break repair by nonhomologous end joining was present in 6\% $(n=26)$ of the whole cohort. The large cohort analysis of tumors as part of PCAWG revealed that ID8 activity was correlated with age at diagnosis, suggesting a clock-like behavior of this signature ${ }^{14}$. However, we did not observe 
214 such correlation in our cohort, potentially due to the very low number of tumors with ID8

215 activity. ID12, a signature with unknown etiology was identified in a small fraction of high-

216 grade glioma and Group3 medulloblastoma. Manual review of aligned reads and variant calls

217 of ID12 assigned indels showed that these mutations were mostly present in low-mappability,

218 repeat-rich regions of the genome, leading to the hypothesis that ID12 might present an

219 artefact signature in this cohort at least.

220

221 Next, we sought to identify potential associations of indel signature activity with age, TP53

222 mutation status, chromothripsis and genomic instability. Of the 9 ID signatures active in our

223 cohort, we observed that ID1 and ID2 signature activity is highly correlated with age at

224 diagnosis (Supp. Fig. 4a), revealing their clock-like behavior in pediatric cancers as observed

225 in adult cancers ${ }^{14}$. The number of mutations attributed to ID2 and ID9 (of unknown etiology)

226 were significantly different depending on the TP53 mutation status. TP53 germline/somatic

227 mutated tumors showed higher activity of ID2 and ID9 compared with wildtype tumors

228 (Supp. Fig 4b). Similarly, a significantly higher number of mutations were attributed to ID2

229 and ID9 in chromothriptic tumors compared to non-chromothriptic tumors (Supp. Fig. 4c). A

230 similar difference was observed in adult cancers ${ }^{25}$. The total number of structural variants was

231 highly correlated with ID9 signature activity across the cohort. A per-cancer type analysis

232 revealed that ID9 and genomic instability were highly correlated in RMS (Supp. Fig. 4d). In

233 addition, ID3 and ID4 activities were observed in a small fraction of tumors (Supp. Table 8),

234 however, we did not identify any common genomic alterations across these tumors.

235

236 Finally, we performed a correlation analysis of SBS and ID signature activities across the

237 cohort to understand if any of the SBS and ID signatures co-occurred. We observed a high

238 correlation amongst clock-like signatures SBS1, SBS5 and ID1, ID2 (Fig. 2c). In addition,

239 ID3, a tobacco smoking related signature was highly correlated with SBS31 and SBS35, both

240 of which are due to DNA damage caused by platinum treatment (Fig. 2b, c).

241

242 In summary, we extracted 27 SBS signatures and 9 ID signatures in our pediatric pan-cancer

243 cohort that overlap COSMIC v.3 signatures. The total number of identified signatures is lower

244 than those identified in adult cancers ${ }^{14}$ and a large fraction of mutations (57\% and $42 \%$ of

245 SBS mutations; 85\% and 100\% of ID mutations in non-hyper mutated and hyper-mutated

246 samples, respectively) were attributed to clock-like signatures such as SBS1, SBS5, ID1 and 
247 ID2. A significant difference in terms of mutations attributed to SBS2, SBS8 and SBS13 as

248 well as ID2 and ID9 was observed to be depending on TP53 mutation status.

250 Homologous recombination repair defect signature activities

251 The homologous recombination (HR) repair pathway is an error-free mechanism to repair 252 DNA double-strand breaks ${ }^{26}$. Genomic alterations in components of the HR pathway, mainly 253 in the BRCA1/2 genes, lead to a characteristic pattern of single base substitution mutations 254 and large deletions at microhomology regions, a phenotype frequently termed 'BRCAness, 27. 255 Previous studies focusing mainly on breast and ovarian cancers have identified a strong 256 correlation between BRCA1/2 biallelic pathogenic mutations and activity of signature 3 257 (COSMIC v.2). However, a significant fraction of these tumors showed signature 3 activity 258 without any identifiable alterations in HR pathway components ${ }^{5,11,28,14}$. Discerning the 259 activity of HR defect COSMIC v.3 signatures SBS3 and ID6 in tumors is important as it has 260 previously been shown to be associated with the therapeutic response to platinum and poly 261 ADP-ribose polymerase (PARP) inhibitor treatment ${ }^{29,12,30,31,27,28,46}$.

262

263 Previous analyses of mutational signatures in pediatric cancers, including our own published 264 analysis $^{2}$, have identified a significant proportion of Signature.3 (COSMIC v.2), mostly in 265 tumors without any HR pathway gene defects ${ }^{2}$. However, the current analysis with COSMIC 266 v.3 signatures identified only a small fraction (2.23\% of the whole cohort) of tumors with 267 SBS3 signature activity (Fig. 3a,b). This marked difference is most likely the result of 268 previous Signature 3 mutations now being attributed to “flat" signatures (e.g., SBS5 and 269 SBS40) of the updated and refined COSMIC v.3 mutational signatures. In addition, there is a 270 difference in the approach compared to the initial signature analysis, as we assume SBS1 and 271 SBS5 as background signatures and only add SBS3 if it improves the cosine similarity with at 272 least 0.02. However, none of these tumors with SBS3 showed the associated ID6 signature 273 activity, represented by a high fraction of long deletions at microhomology regions (Fig. 2a, 274 Fig. 3b). This lack of ID6 prompted us to test whether our current variant calling pipeline 275 could be penalizing deletions at microhomologies by assigning low confidence, for example, 276 or if pediatric cancers in general have very low numbers of microhomology-associated 277 deletions as an inherent property. In order to test this further, we whole-genome sequenced 278 five tumors from the INFORM registry ${ }^{32}$ with known (likely) pathogenic germline mutations 279 in BRCA1/2 based on ClinVar. Amongst these, INF_R_1076 had a BRCA2 pathogenic 280 homozygous mutation and INF_R_025 a BRCA2 compound heterozygous mutation (both 
281 patients also had a clear phenotype, i.e. Fanconi anaemia), while the remaining tumors had 282 heterozygous (likely) pathogenic BRCA1/2 mutations (Fig. 3c) without any second hit in the 283 tumor. Mutational signature analysis of these five pediatric tumors revealed that only the two 284 tumors with compound heterozygous and homozygous BRCA2 mutations, i.e. biallelic 285 inactivation, showed SBS3 and ID6 signature activity (Fig. 3c, Supp. Table 10). In addition, 286 we analyzed 22 whole genome sequenced adult tumors from the NCT-MASTER precision 287 oncology program (https://www.nct-heidelberg.de/master) with respect to ID signatures, for 288 which somatic mutations were called with the same DKFZ in-house pipeline. Amongst these, 289 half of the tumors $(n=11)$ had known BRCA1/2 deficiency and showed clear ID6 activity 290 (Supp. Fig. 5a, Supp. Table 11). These results indicate that our variant calling pipeline does 291 not systematically miss the deletions at microhomology regions and confirm the importance 292 of biallelic BRCA1/2 inactivation for the presence of predictive HR defect-associated 293 mutational signatures, that is SBS3 and ID6.

294 In the Gröbner et al. publication ${ }^{2}$, the initial analysis of this pediatric pan-cancer cohort, six 295 patients were identified with a pathogenic/likely pathogenic BRCA2 germline variant (Supp. 296 Table 13). Five of these patients were heterozygous in the germline and without evidence for 297 biallelic inactivation in the tumor, while one case was compound heterozygous in the 298 germline for BRCA2 (SJMB012, 8 year old male with SHH-medulloblastoma). For the latter 299 case, one BRCA2 germline variant (ENSP00000439902.1:p.Val2407GlufsTer60) is not 300 currently reported in ClinVar and the other one as likely pathogenic (variationID: 421014). We did not identify SBS3 or ID6 activity in any of these six tumors.

302

303 Next, we sought to understand whether a proportion of long deletions ( $>5 \mathrm{bp}$ ) at 304 microhomologies ( $\mathrm{MH}$ ) are present in pediatric tumors, albeit at very low levels. In order to 305 understand how MH-associated deletions are represented in ID6 positive adult tumors, we 306 divided the PCAWG whole genome tumors $(n=2,776)$ into different categories depending on 307 the presence of SBS3 and ID6. Then we compared the proportion of MH-associated deletions 308 of tumors in these categories with our pediatric cancer cohort. This analysis revealed that 309 pediatric tumors overall have very few MH-associated deletions compared with adult tumors 310 and a significantly lower fraction compared with ID6 positive tumors (Fig. 3d). However, in 311 our cohort, one osteosarcoma tumor (SJOS004) showed ID6 signature without any activity of 312 the SBS3 signature (Fig. 3e). Amongst its SBS signatures, a high fraction of SBS40 mutations 313 were observed. SBS3 and SBS40 are relatively flat signatures and have a similar feature 314 distribution (cosine similarity of 0.88 ). For this reason, the possibility of mis-assigning SBS3 
315 mutations to SBS40 cannot be excluded. However, no HR pathway mutations were identified

316 in this osteosarcoma tumor.

317

318 Amongst the tumors with SBS3 signature activity in our cohort, 58\% (7/12) belong to the

319 Group3 subgroup of medulloblastoma (MB). MB tumors are known to follow a linear 320 increase of somatic mutation burden with age ${ }^{33}$. Presumably, mutations in tumors that do not

321 follow such a correlation could be contributed by mutational processes other than the 322 ubiquitous clock-like signatures. In this analysis, we identified only one tumor (SJMB008) 323 with an active SBS3 signature as an outlier of age versus mutation burden correlation, 324 suggesting that most of the other SBS3-positive Group3 MBs follow the typical linear 325 increase in mutation burden with age (Supp. Fig. 5b), and therefore the possibility of mis326 assigning mutations from clock-like signatures (especially SBS5 and SBS40) to SBS3 cannot 327 be excluded. Next, we compared expression of HR pathway genes such as BRCA1/2 and 328 PALB2 between SBS3 positive and negative tumors in Group $3 \mathrm{MBs}$, as there could be other 329 mechanisms acting to inactivate the expression of these genes. We observed only a slight 330 difference in PALB2 expression (Supp. Fig. 5c), but not in the other genes and also no 331 significant difference in promoter DNA methylation of these genes (data not shown).

333 In summary, we identified only a small percentage (2.23\%) of pediatric tumors in our cohort 334 with SBS3 (COSMIC v.3) activity, the so-called HR deficient phenotype, compared to 54\% 335 of tumors with Signature 3 (COSMIC v.2) activity in our previous analysis of the same 336 cohort $^{2}$. Furthermore, in tumors with SBS3 signature activity, we could not identify any 337 genomic alterations or loss of expression of HR pathway genes. The genome of these SBS3 338 positive tumors was relatively stable with very few outliers (Supp. Fig. 6). The indel signature 339 ID6, which is characterized by long deletions (>5bp) at microhomology sequences, is a strong 340 predictor of HR deficiency and was not identified in our cohort except for one osteosarcoma 341 tumor. Further analysis of $n=5$ INFORM and $n=22$ NCT-MASTER whole genome sequenced 342 tumors suggests the importance of genetic biallelic BRCA1/2 inactivating events in the generation of HR deficient mutational signatures.

345 Signature.P1 similarity to COSMIC v.3 signatures

346 Previous mutational signature analysis of this cohort based on the COSMIC v.2 reference 347 signatures identified a novel substitution signature, called Signature P1, which featured 348 elevated mutations of $\mathrm{C}>\mathrm{T}$ in the context of CCC/CCT ${ }^{2}$. Signature P1 was active in the 
349 pediatric brain tumors atypical teratoid/rhabdoid tumors (ATRT) and ependymoma. In the 350 current analysis, the Signature P1 profile was compared to all identified COSMIC v.3 SBS 351 signatures and a high similarity was observed with signature SBS31 (Fig. 4a,b). SBS31 is the

352 result of DNA damage caused by platinum treatment. In the present analysis, SBS31 activity 353 was identified in ATRT, ependymoma and ETMR tumors (Fig. 4c). These results suggest that 354 Signature.P1 is not, as previously hypothesized, a pediatric specific mutational signature, but 355 rather a treatment-associated signature identified in a small fraction of tumors that were 356 annotated as treatment naïve. These patients had likely been treated prior to genomic analysis, 357 and at least for one ATRT sample (H049-JVCT; high SBS35 activity, Suppl. Table 2) we 358 could follow up with the sample source which confirmed that this tumor was a recurrence and 359 not a primary tumor.

360 
361

362

363

364

365

366

367

368

369

370

371

372

373

374

375

376

377

378

379

380

381

382

383

384

385

386

387

388

389

390

391

392

393

394

\section{Discussion}

In this study, we re-analyzed 537 whole genome sequenced tumor-normal pairs from 20 different molecularly defined entities of childhood cancers to identify and refine the mutational signatures of the underlying mutational processes. We examined single base substitution (SBS) and small insertion/deletion (ID) in depth and showed that a relatively small number of mutational processes operate in pediatric cancers compared with adult cancers. Amongst the identified 27 SBS and 9 ID signatures, etiologies for more than half of these signatures have been described either by experimental approaches or association analyses in previous studies in adult cancers. In this cohort, a large fraction of SBS (45.4\%) and ID (93.2\%) mutations across multiple cancer types were attributed to clock-like substitution signatures SBS1 and SBS5,as well as to clock-like indel signatures. Signatures such as SBS8 are relatively frequent across cancer entities, but the etiology remains unknown and recent evidence suggests this signature is due to DNA damage induced by late replication errors $^{24}$. Other signatures without known etiology were identified in a small fraction of tumors from specific cancer types such as pilocytic astrocytoma (namely, SBS12 and SBS23) and Burkitt's lymphoma (namely, SBS17a, SBS17b). However, we did not identify any genomic alteration common to these tumors.

Previous mutational signature analyses identified Signature 3 (equivalent to the current SBS3) along with other features, with or without discernable alterations in BRCA1/2 genes, as a potential biomarker for HR deficiency and platinum plus PARPi-based treatment response $^{34,35}$. In pediatric cancers, germline predisposition or somatic alterations in BRCA1/2 genes are infrequent ${ }^{2,3}$ and only a small fraction (2.23\%) of tumors showed SBS3 activity in this analysis, but without the complementing indel signature ID6. Microhomology (MH)associated deletions, a strong feature of ID6, were significantly more rare in pediatric than in adult cancers. A plausible explanation for this observation could be that the total somatic mutation burden of childhood cancers is very $l_{0}{ }^{2}$ and the likelihood of mutations occurring at $\mathrm{MH}$ sequences will therefore also be small. Polak et al. ${ }^{11}$ identified a signature that is similar to the current SBS3 in breast cancer tumors without alterations in BRCA1/2 genes, but identified alterations in other HR pathway components (e.g. PALB2 or RAD51C). In this pediatric cancer cohort, we have not identified genomic alterations in other HR pathway genes that could potentially result in the observed SBS3 signature. While there might be other epigenetic mechanisms to inactivate HR pathway genes, in Group3 medulloblastoma (for which complementary omics data is available ${ }^{33,47}$ ) we have not observed promoter 
395 hypermethylation or reduced expression of HR pathway genes. Furthermore, we do not 396 discount the possibility of mis-assigning SBS5 and SBS40 signature mutations to SBS3. In 397 addition, a recent study suggests that SBS3 is likely not as specific as previously believed and 398 that the identification of HR deficiency should rely on multiple orthogonal mutational 399 signatures, not only on $\mathrm{SBS}^{48}$. In order to validate the biomarker efficacy of SBS3 to predict 400 PARP inhibitor treatment response in pediatric cancers, experiments involving PDX models 401 of pediatric tumors are currently ongoing (for example in the "BRCAddict" project, 402 https://www.transcanfp7.eu/index.php/abstract/brcaddict.html). Recent studies focusing on 403 Ewing sarcoma ${ }^{37}$ and ETMR brain tumors ${ }^{38}$ identified the presence of R-loops, DNA-RNA 404 hybrid structures, to be correlated with PARP inhibitor response. In the future, mutational 405 signature SBS3 combined with other features such as indel signature ID6 and/or R-loops 406 should be investigated in preclinical models to asses PARP inhibitor response and to 407 ultimately define optimized biomarkers.

408

409 In summary, although some cancer entities are under-represented in this cohort, we believe 410 this analysis on the mutational signature repertoire in childhood cancers provides a valuable

411 resource for further understanding of tumor biology and aids future research in defining 412 biomarkers of treatment response.

413 


\section{METHODS}

415

\section{Whole-genome sequencing data, alignment, and variant calling}

417 All whole genome sequencing data analyzed in this study was collected from Gröbner, Worst 418 et al., 2018². Briefly, FASTQ data was aligned to reference genome GRCh37/hg19 with 419 BWA-MEM (v 0.7.8) ${ }^{39}$. Single base substitutions were called using an updated samtools ${ }^{40}$ 420 based DKFZ in-house pipeline (0.1.19) and indels were called using Platypus (0.8.1.1).

421

\section{Somatic Mutation Frequencies}

423 Single base substitution and small insertion/deletion mutation burden was calculated as the 424 total number of mutations identified per megabase of the genome. For whole genome 425 sequencing data, the total number of mutations was divided by 2800 (effective human genome 426 size in megabases that can be assessed by whole-genome sequencing).

\section{Analysis of mutational signatures using SigProfiler}

429 In order to extract mutational signatures, 96-context SBS and 83-context ID mutational catalogues were prepared using SigProfilerMatrixGenerator (version 1.0.24) ${ }^{41}$ for each of the 20 cancer types. These mutational catalogues were used as input to SigProfilerExtractor (version 1.0.19) ${ }^{42}$ to extract signatures and attribute mutations to each signature in every individual tumor. Briefly, SigProfilerExtractor applies a nonnegative matrix factorization algorithm in multiple iterations $(n=100)$ and for each iteration the software minimizes a generalized Kullback-Leibler divergence constrained for non-negativity. The optimal number of mutational signatures was selected based on highest average stability and lowest average sample cosine distance. After the optimal number of signatures was estimated, attribution of mutations to each signature in each sample involved finding the minimum of the Frobenius norm of a constrained function using a nonlinear convex optimization programming solver based on the interior point algorithm ${ }^{21}$.

\section{Structural variants and copy-number profiles}

443 Structural variants per tumor were identified as described previously using the DELLY ${ }^{43}$ 444 ICGC pan-cancer analysis workflow (https://github.com/ICGC-TCGA445 PanCancer/pcawg_delly_workflow). Copy-numbers were estimated using the ACEseq (allele446 specific copy-number estimation from sequencing) too, based on binned tumor-control 447 coverage ratio and B-allele frequencies (BAF) ${ }^{44}$. 
Statistical analyses and code availability

450 All downstream statistical analyses were performed using the $\mathrm{R}$ statistical programming

451 language (version 4.0.3) and all code is available at

452 https://github.com/KiTZ-Heidelberg/Signatures-Manuscript

453

454

455

456

457

\section{Acknowledgements}

This project was supported by funding from the ADDRess consortium (Grant 01GM1909E, BMBF). L.B.A. is an Abeloff $\mathrm{V}$ scholar and he is personally supported by a Packard Fellowship for Science and Engineering. We acknowledge the DKFZ's ODCF and GPCF core facilities for supporting the genomic sequencing and data processing.

462

\section{Contributions}

464 V.T., S.M.P. and N.J. conceptualized the study. S.M.A.I. and L.B.A. contributed to software 465 development and implementation. Analyses were performed by V.T., N.J., G.W., S.M.A.I and 466 L.B.A. Data visualization was conducted by V.T. Data were collected and curation of data 467 was conducted by V.T., B.C.J., S.N.G., B.H., D.H., S.F., M.B.-J., and D.T.W.J. The original 468 draft was written by V.T. and N.J., and all co-authors reviewed and edited the manuscript.

\section{Corresponding authors}

471 Correspondence to Stefan Pfister and Natalie Jäger.

472

473 


\section{REFERENCES}

476

477 1. Pui, C., Gajjar, A. et al. Challenging issues in pediatric oncology. Nat. Rev. Clin. Oncol 540, 540-549

$478 \quad$ (2011).

479 2. Gröbner, S., Worst, B., Weischenfeldt, J. et al. The landscape of genomic alterations across childhood

$480 \quad$ cancers. Nature 555, 321-327 (2018)

481 3. Ma, X. et al. Pan-cancer genome and transcriptome analyses of 1,699 paediatric leukaemias and solid

$482 \quad$ tumours. Nature 555, 371-376 (2018).

483 4. Stratton, M. R., Campbell, P. J. \& Futreal, \& P. A. The cancer genome. Nature 458, (2009).

484 5. Alexandrov, L., Nik-Zainal, S., Wedge, D. et al. Signatures of mutational processes in human cancer.

$485 \quad$ Nature 500, 415-421 (2013)

486 6. Nik-Zainal, S. et al. Mutational processes molding the genomes of 21 breast cancers. Cell 149, 979-993

487 (2012).

488 7. Alexandrov, L., Jones, P., Wedge, D. et al. Clock-like mutational processes in human somatic cells. Nat

$489 \quad$ Genet 47, 1402-1407 (2015).

490 8. Schulze, K., Imbeaud, S., Letouzé, E. et al. Exome sequencing of hepatocellular carcinomas identifies

491 new mutational signatures and potential therapeutic targets. Nat Genet 47, 505-511 (2015).

492 9. Nik-Zainal, S. et al. Landscape of somatic mutations in 560 breast cancer whole-genome sequences.

$493 \quad$ Nature 534, 47-54 (2016).

494 10. Petljak, M. \& Alexandrov, L. B. Understanding mutagenesis through delineation of mutational

495 signatures in human cancer. Carcinogenesis 37, 531-540 (2016).

496 11. Polak, P. et al. A mutational signature reveals alterations underlying deficient homologous

497 recombination repair in breast cancer. Nat. Genet. 49, 21 (2017).

498 12. Keung, M., Wu, Y. \& Vadgama, J. PARP Inhibitors as a Therapeutic Agent for Homologous

499 Recombination Deficiency in Breast Cancers. J. Clin. Med. 8, 435 (2019).

500 13. Campbell, P. J. et al. Pan-cancer analysis of whole genomes. Nature 578, 82-93 (2020).

501 14. Alexandrov, L. B. et al. The repertoire of mutational signatures in human cancer. Nature 578, 94-101

$502 \quad$ (2020).

503 15. Johann, P. D. et al. Atypical Teratoid/Rhabdoid Tumors Are Comprised of Three Epigenetic Subgroups

$504 \quad$ with Distinct Enhancer Landscapes. Cancer Cell 29, 379-393 (2016).

505 16. Northcott, P., Buchhalter, I., Morrissy, A. et al. The whole-genome landscape of medulloblastoma

$506 \quad$ subtypes. Nature 547, 311-317 (2017)

507 17. Kovac, M. et al. Exome sequencing of osteosarcoma reveals mutation signatures reminiscent of BRCA

508 deficiency. Nat. Commun. 6, 1-9 (2015).

509 18. Kool, M. et al. Genome sequencing of SHH medulloblastoma predicts genotype-related response to

$510 \quad$ smoothened inhibition. Cancer Cell 25, 393-405 (2014).

511 19. Kunz, J. B. et al. Pediatric T-cell lymphoblastic leukemia evolves into relapse by clonal selection,

512 acquisition of mutations and promoter hypomethylation. Haematologica 100, 1442-1450 (2015).

513 20. Jones, D. T. W. et al. Recurrent somatic alterations of FGFR1 and NTRK2 in pilocytic astrocytoma. Nat.

$514 \quad$ Genet. 45, 927-932 (2013). 
515 21. Alexandrov, L. B., Nik-Zainal, S., Wedge, D. C., Campbell, P. J. \& Stratton, M. R. Deciphering

516

517

518

519

520

521

522

523

524

525

526

527

528

529

530

531

532

533

534

535

536

537

538

539

540

541

542

543

544

545

546

547

548

549

550

551

552

553

554

555 Signatures of Mutational Processes Operative in Human Cancer. Cell Rep. 3, 246-259 (2013).

22. Periyasamy, M. et al. p53 controls expression of the DNA deaminase APOBEC3B to limit its potential mutagenic activity in cancer cells. Nucleic Acids Res. 45, 11056-11069 (2017).

23. Wang, S., Jia, M., He, Z. \& Liu, X.-S. APOBEC3B and APOBEC mutational signature as potential predictive markers for immunotherapy response in non-small cell lung cancer. Oncogene 37, 3924-3936 (2018).

24. Singh, V.K., Rastogi, A., Hu, X. et al. Mutational signature SBS8 predominantly arises due to late replication errors in cancer. Commun Biol 3, 421 (2020).

25. Voronina, N., Wong, J.K.L., Hübschmann, D. et al. The landscape of chromothripsis across adult cancer types. Nat Commun 11, 2320 (2020).

26. Jasin, M. \& Rothstein, R. Repair of Strand Breaks by Homologous Recombination. (2013) doi:10.1101/cshperspect.a012740

27. Byrum, A. K., Vindigni, A. \& Mosammaparast, N. Defining and Modulating 'BRCAness'. Trends in Cell Biology 29, 740-751 (2019).

28. Davies, H. et al. HRDetect is a predictor of BRCA1 and BRCA2 deficiency based on mutational signatures. Nat. Med. 23, 517-525 (2017).

29. Póti, Á. et al. Correlation of homologous recombination deficiency induced mutational signatures with sensitivity to PARP inhibitors and cytotoxic agents. Genome Biol. 20, 240 (2019).

30. Chen, A. PARP inhibitors: its role in treatment of cancer. Chinese journal of cancer 30, 463-471 (2011).

31. Tutt, A. et al. Oral poly(ADP-ribose) polymerase inhibitor olaparib in patients with BRCA1 or BRCA2 mutations and advanced breast cancer: A proof-of-concept trial. Lancet 376, 235-244 (2010).

32. Worst, B. C. et al. Next-generation personalised medicine for high-risk paediatric cancer patients - The INFORM pilot study. Eur. J. Cancer 65, 91-101 (2016).

33. Jones, D. T. W. et al. Dissecting the genomic complexity underlying medulloblastoma. Nature 488, 100-105 (2012).

34. Ma, J., Setton, J., Lee, N. Y., Riaz, N. \& Powell, S. N. The therapeutic significance of mutational signatures from DNA repair deficiency in cancer. Nature Communications 9, (2018).

35. Zhao, E. Y. et al. Personalized Medicine and Imaging Homologous Recombination Deficiency and Platinum-Based Therapy Outcomes in Advanced Breast Cancer. Clin Cancer Res 23, (2017).

36. Machado, H. et al., Genome-wide mutational signatures of immunological diversification in normal lymphocytes. (2021) bioRxiv 2021.04.29.441939; doi: https://doi.org/10.1101/2021.04.29.441939

37. Gorthi, A. et al. EWS-FLI1 increases transcription to cause R-Loops and block BRCA1 repair in Ewing sarcoma. Nature 555, 387-391 (2018).

38. Lambo, S. et al. The molecular landscape of ETMR at diagnosis and relapse. Naure 576, 576(7786):274280 (2019).

39. Li, H. \& Durbin, R. Fast and accurate short read alignment with Burrows-Wheeler transform. Bioinformatics 25, 1754-1760 (2009).

40. Li, H. et al. The Sequence Alignment/Map format and SAMtools. Bioinforma. Appl. NOTE 25, 20782079 (2009).

41. Bergstrom, E. N. et al. SigProfilerMatrixGenerator: A tool for visualizing and exploring patterns of 
small mutational events. BMC Genomics 20, 685 (2019).

42. Ashiqul Islam, S. M. et al. Uncovering novel mutational signatures by de novo extraction with. (2021) doi:10.1101/2020.12.13.422570, BioRxiv

43. Rausch, T. et al. DELLY: structural variant discovery by integrated paired-end and split-read analysis. Bioinformatics 28, 333-339 (2012).

44. Kleinheinz, K. et al. ACEseq-allele specific copy number estimation from whole genome sequencing. (2017) doi:10.1101/210807, BioRxiv

45. Li, B. et al., Therapy-induced mutations drive the genomic landscape of relapsed acute lymphoblastic leukemia. Blood. 2020; 135(1): 41-55.

46. Gröschel, S., Hübschmann, D., Raimondi, F. et al. Defective homologous recombination DNA repair as therapeutic target in advanced chordoma. Nat Commun 10, 1635 (2019).

47. Hovestadt, V., Jones, D., Picelli, S. et al. Decoding the regulatory landscape of medulloblastoma using DNA methylation sequencing. Nature 510, 537-541 (2014).

48. Degasperi, A., Amarante, T.D., Czarnecki, J. et al. A practical framework and online tool for mutational signature analyses show intertissue variation and driver dependencies. Nat Cancer 1, 249-263 (2020).

\section{Figure Legends}

Figure 1: Mutation burden of SBSs and small indels (IDs) across 20 pediatric tumor types.

a) Single base substitution (SBS) and small insertion/deletion (ID) mutation burden of the pediatric pan-cancer whole-genome sequenced (PPC-WGS) cohort. The numbers of samples for each tumor type are shown next to the labels. Each dot represents one tumor sample. Tumor types are ordered by the median numbers of single-base substitutions. b) Correlation between SBS and ID mutations per megabase across the cohort.

Figure 2: SBS and ID signature activity across pediatric cancers

a) Example profiles of SBS and ID signatures extracted from the PPC-WGS cohort that are similar to COSMIC v.3 SBS3 and ID6 signatures. b) The number of mutations contributed by each mutational signature to the PPC-WGS tumors. Circle size indicates fraction of tumors with signature activity in a cancer type and the color indicates the median number of mutations per megabase due to a signature in a specific entity. c) Correlation between SBS and ID signature activities.

\section{Figure 3: HR defect signatures activity in pediatric cancers}

a) Fraction of all PPC-WGS cohort samples that show activity of Signature.3 (COSMIC v.2) and SBS3 (COSMIC v.3). b) Normalized SBS and ID signature activities in tumors with SBS3 signature activity c) BRCA1/2 mutation status of 5 INFORM whole genome sequenced tumors and their SBS and ID signature activities (MB_SHH - Sonic hedgehog subgroup of medulloblastoma; HGG - high-grade glioma; DIPG - diffuse intrinsic pontine glioma; RMS rhabdomyosarcoma) d) Comparison of microhomology deletion proportion between PCAWG 
600 and PPC-WGS cohorts e) SBS, ID signature activities, microhomology deletion proportions

601

602

603

604

605

606

607

608

609

610

611

612

613

614

615

616

617

618

619

620

621

622

623

624

625

626

627

628

629

630

631

632

633

634

635

636

637

638

639

640

641

642

643

644

645

646

and genome wide copy-number profile of osteosarcoma tumor SJOS004.

Figure 4: Similarity between Signature.P1 and COSMIC v.3 SBS reference signatures

a) Cosine similarity between novel Signature.P1 from Gröbner, Worst et al., 2018 and COSMIC v.3 SBS signatures. b) 96-context mutational profiles of Signature.P1 and SBS31. c) SBS31 signature activity in the PPC-WGS cohort.

\section{Supp. Figure 1: Correlation of SBS and ID mutations with age at tumor diagnosis}

a) Comparison of number of mutation calls identified between 2018 (in Gröbner, Worst et al., 2018) and the updated 2020 DKFZ in-house pipeline. b) Correlation of SBS and ID mutations with age across the PPC-WGS cohort.

Supp. Figure 2: Normalized mutational signature activities across the cohort per sample a) Normalized SBS signature activities across the cohort b) Normalized ID signature activities

\section{Supp. Figure 3: Association of SBS signature activities and genomic alterations}

a) Correlation of SBS1, SBS5 and SBS40 signature activities and age at diagnosis. b) Association of TP53 mutation status and SBS2 and SBS13 signature activities c) Difference in number of CC>TT double base substitutions between SBS7a/b positive and negative tumors of B-ALL-HYPO tumors. d) Association of TP53 mutation status and SBS8 signature activity e) Number of IGHV mutations and SBS9 signature activities in Burkitt's lymphoma samples.

f) Profile of the novel SBS signature identified in Group4-subgroup of medulloblastoma (Group4MB-SBS96D) g) Association of chromothripsis and SBS8 and SBS40 signature activities. h) Association of MYCN-amplification status and SBS18 signature activity i) Correlation of genomic instability and SBS2, SBS13 and SBS40 signature activities.

\section{Supp. Figure 4: Association of ID signature activities and genomic alterations}

a) Correlation of ID1, ID2, ID5 signature activities and age at diagnosis b) Association of TP53 mutation status and ID1, ID2 and ID9 signature activities c) Association of chromothripsis and ID2, ID9 signature activities d) Correlation of genomic instability and ID9 signature activity

\section{Supp. Figure 5: ID signatures in adult cohort and downstream analysis of SBS3 positive} tumors

a) ID signature activities in adult tumors of NCT-MASTER b) Correlation of SBS mutation burden and age at diagnosis in medulloblastoma c) HR pathway gene expression difference between SBS3 positive and negative tumors of Group3 subgroup of medulloblastoma d) HR pathway gene expression in INFORM whole genome sequenced tumors

Supp. Figure 6: Genome-wide copy-number profiles of SBS3 positive tumors from the PedPanCan cohort and INFORM tumors $(n=5)$

TCN $=$ Tumor Copy Number. Red indicates loss of genomic material, green indicates gains. Plots were generated using the ACEseq tool. 


\section{Fig. 1: Tumor mutation burden of pediatric cancers}

a

PPC-WGS cohort $(n=537)$

b

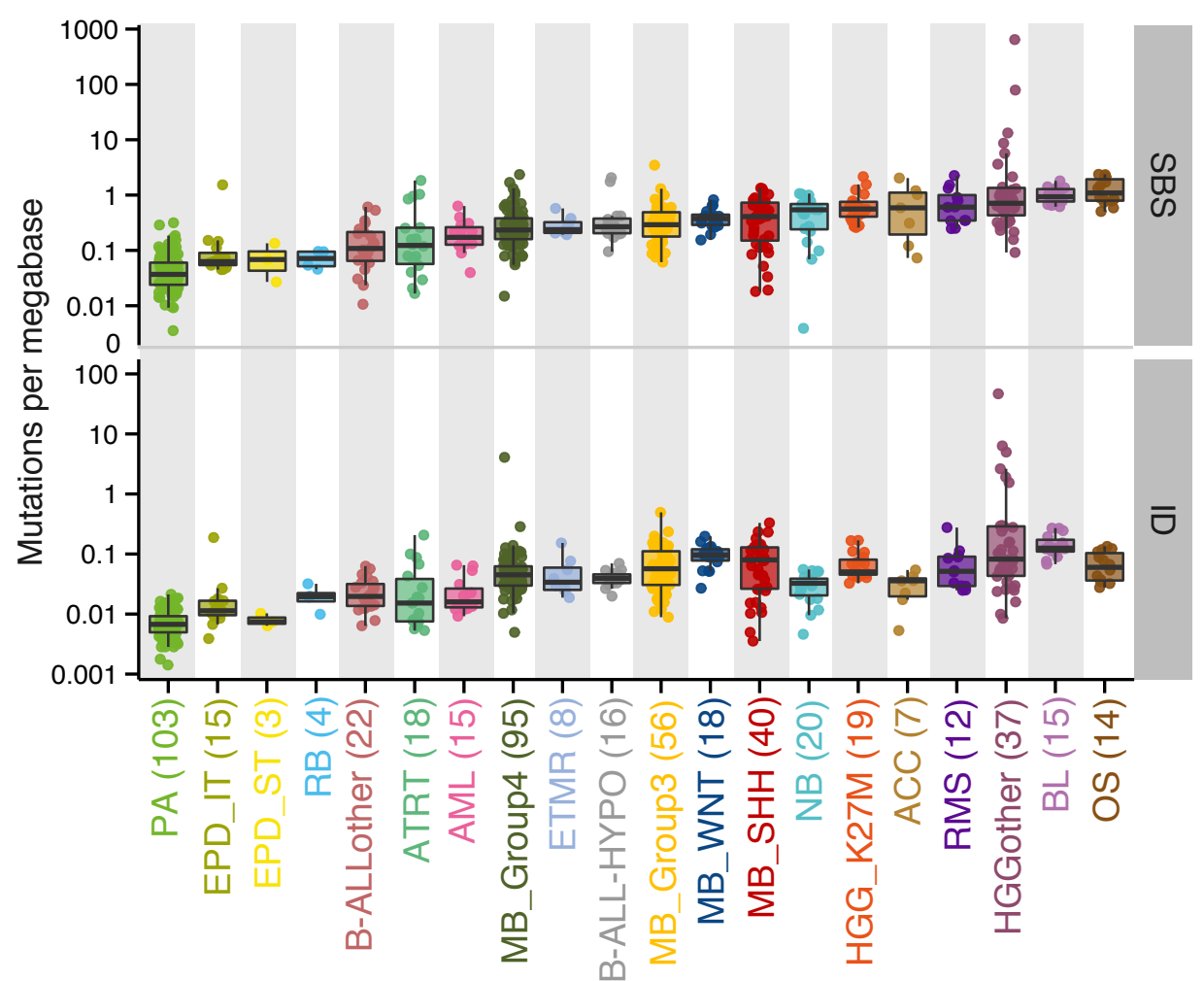

ACC, Adrenocortical carcinoma

AML, Acute myeloid leukemia

ATRT, Atypical teratoid rhabdoid tumors

B-ALL-HYPO, B-cell acute lymphoblastic leukemia, hypodiploid

B-ALLother, B-cell acute lymphoblastic leukemia, non-hypodiploid

BL, Burkitt's lymphoma

EPD_IT, Ependymoma infratentorial

EPD-ST, Ependymoma supratentorial

ETMR, Embryonal tumors with multilayered rosettes

HGG_K27M, High-grade glioma K27M

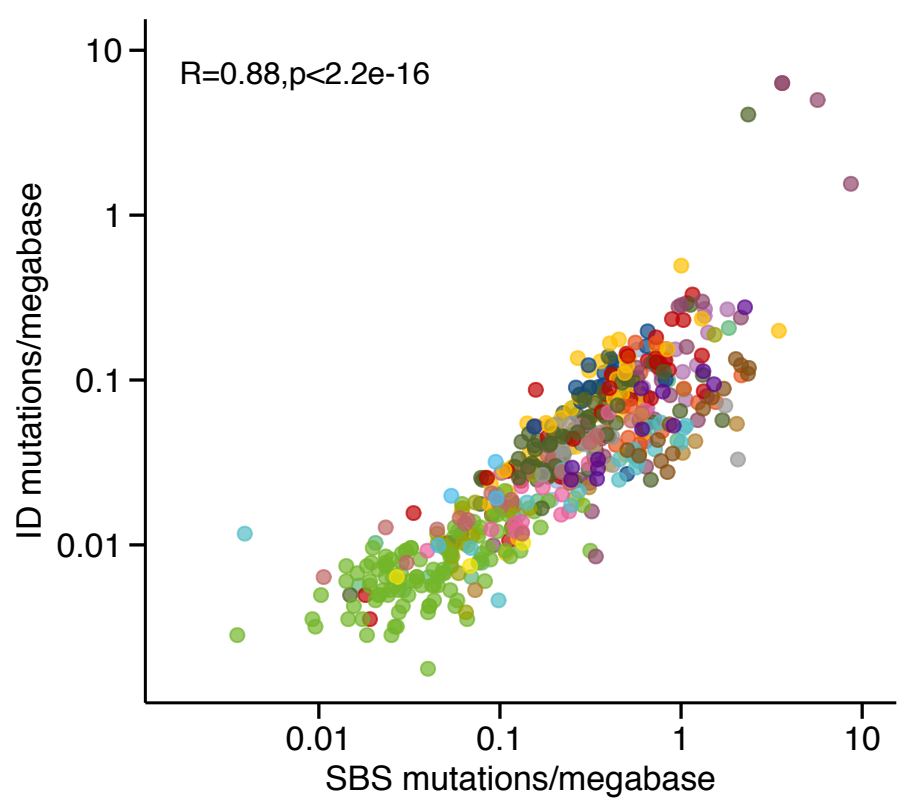

HGGother, High-grade glioma K27wt

- MB_Group3, Medulloblastoma Group3

MB_Group4, Medulloblastoma Group4

MB_SHH, Medulloblastoma SHH

MB WNT, Medulloblastoma WNT

NB, Neuroblastoma

OS, Osteosarcoma

PA, Pilocytic astrocytoma

RB, Retinoblastoma

RMS, Rhabdomyosarcoma 


\section{Fig. 2: SBS and ID signatures in pediatric cancers} a

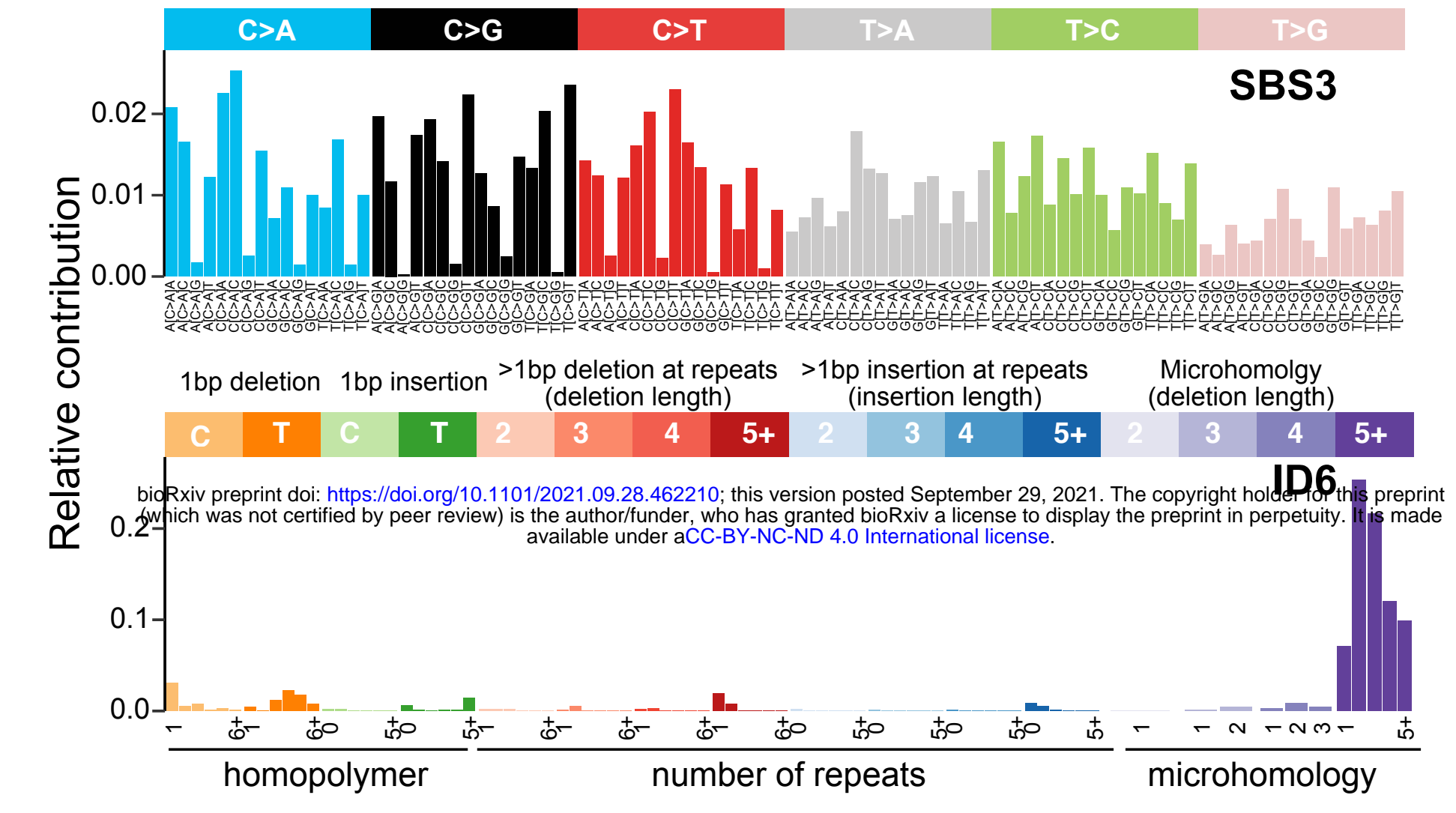

C

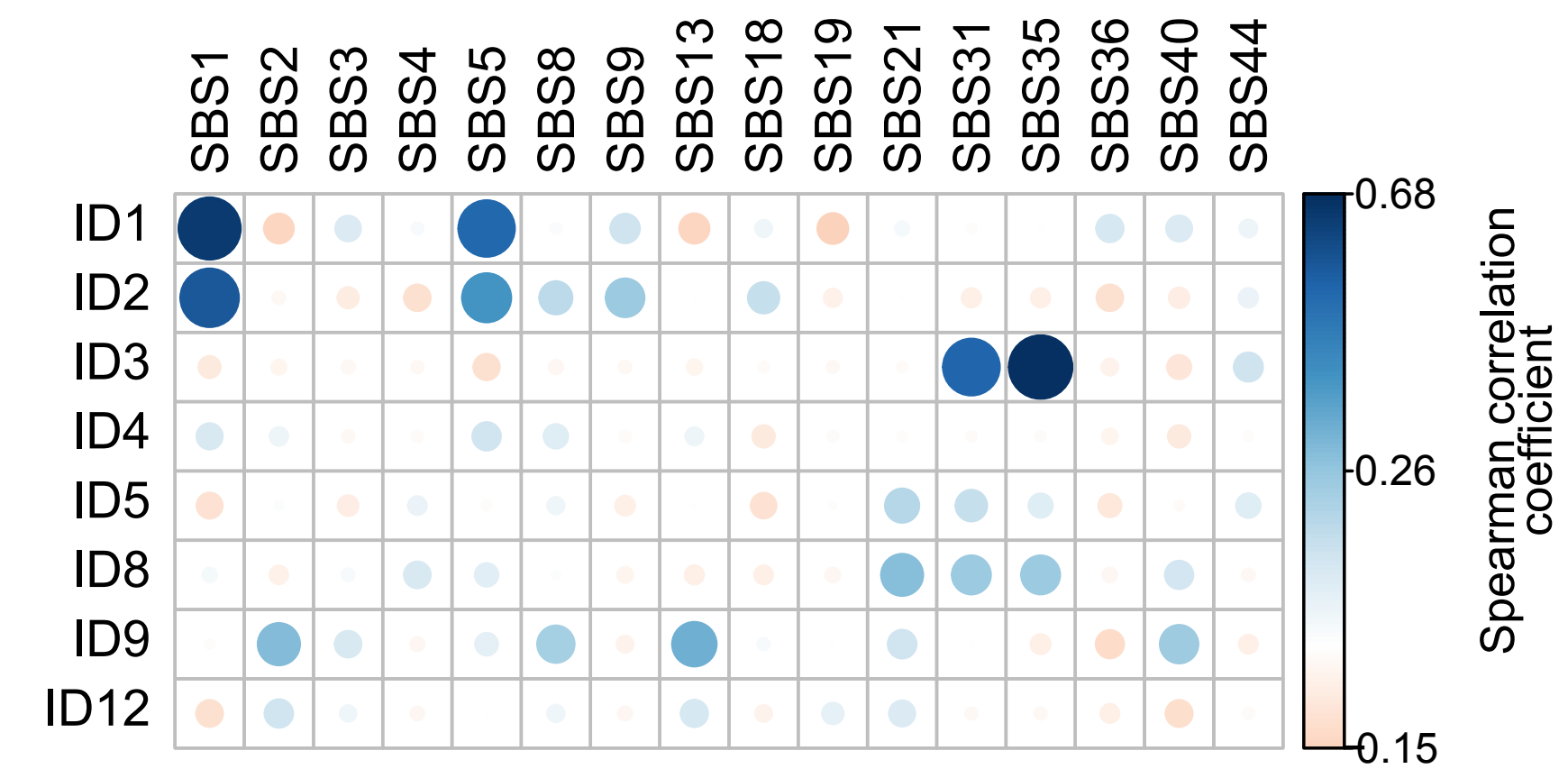

b Proportion of tumors

Median mutations/Mb due to signature

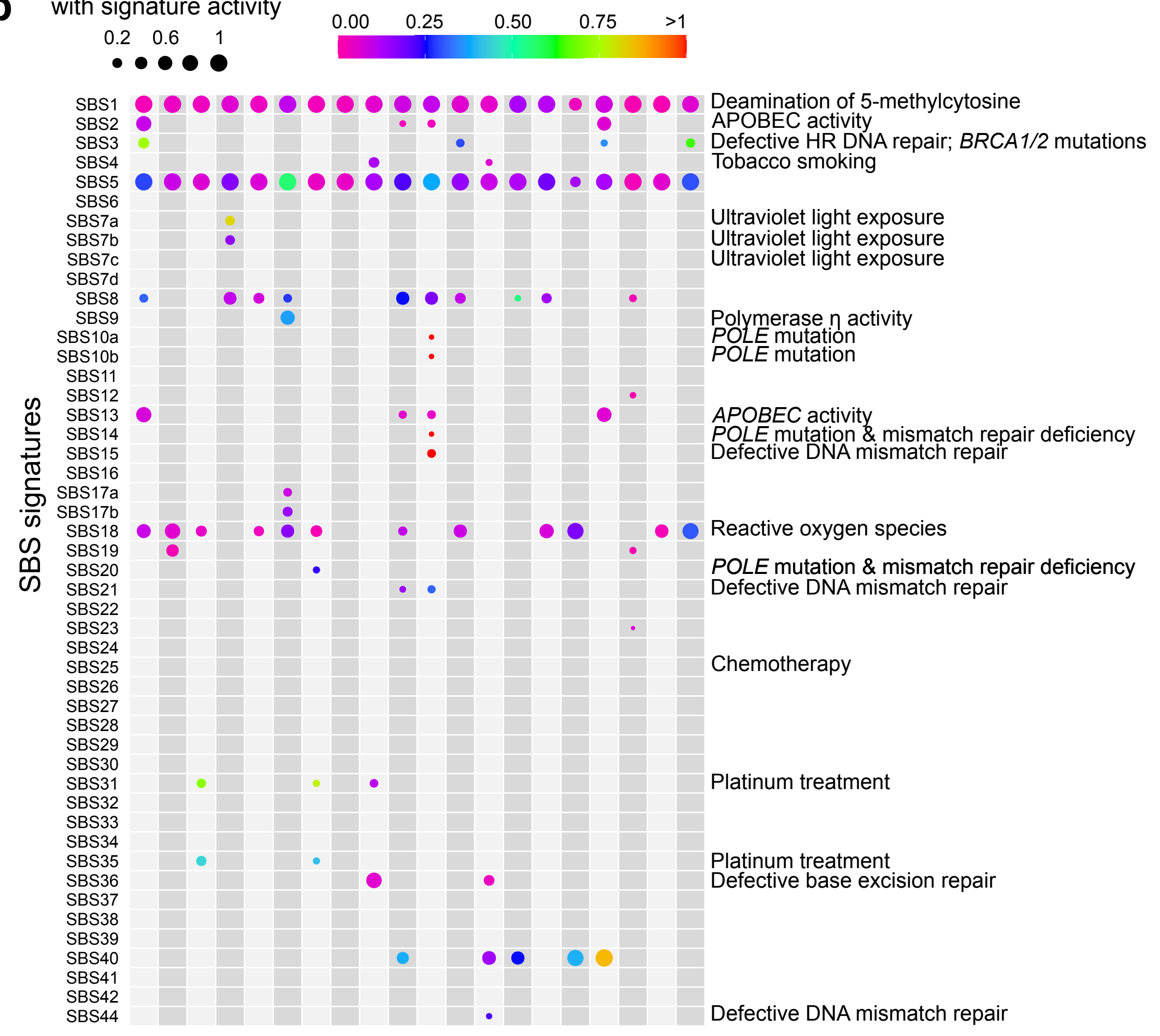

000000000000 Replication slippage/defective DNA mismatch repair ID2 Replication slippage/defective DNA mismatch repair

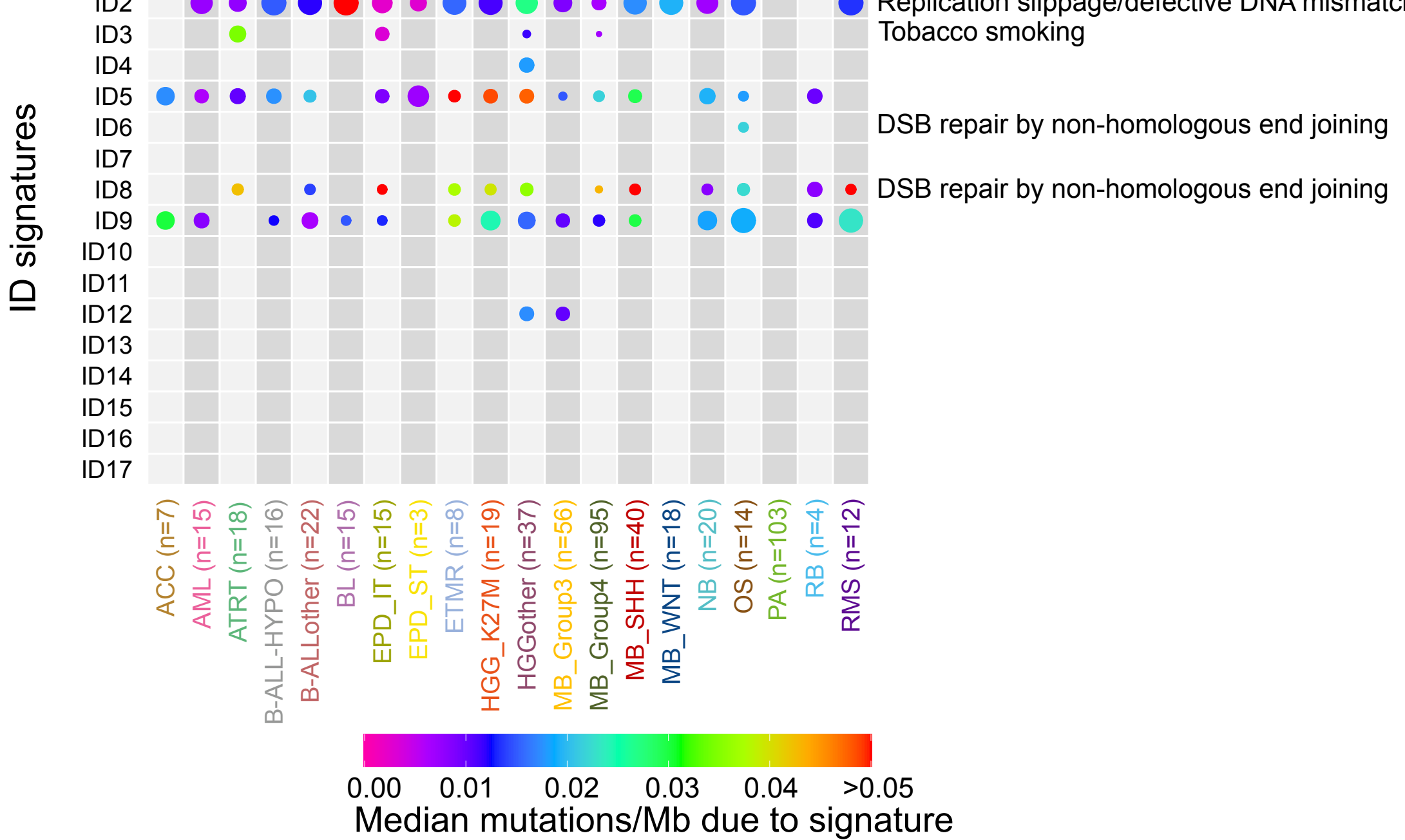


Fig. 4: Similarity between Signature.P1 and SBS reference signatures

a
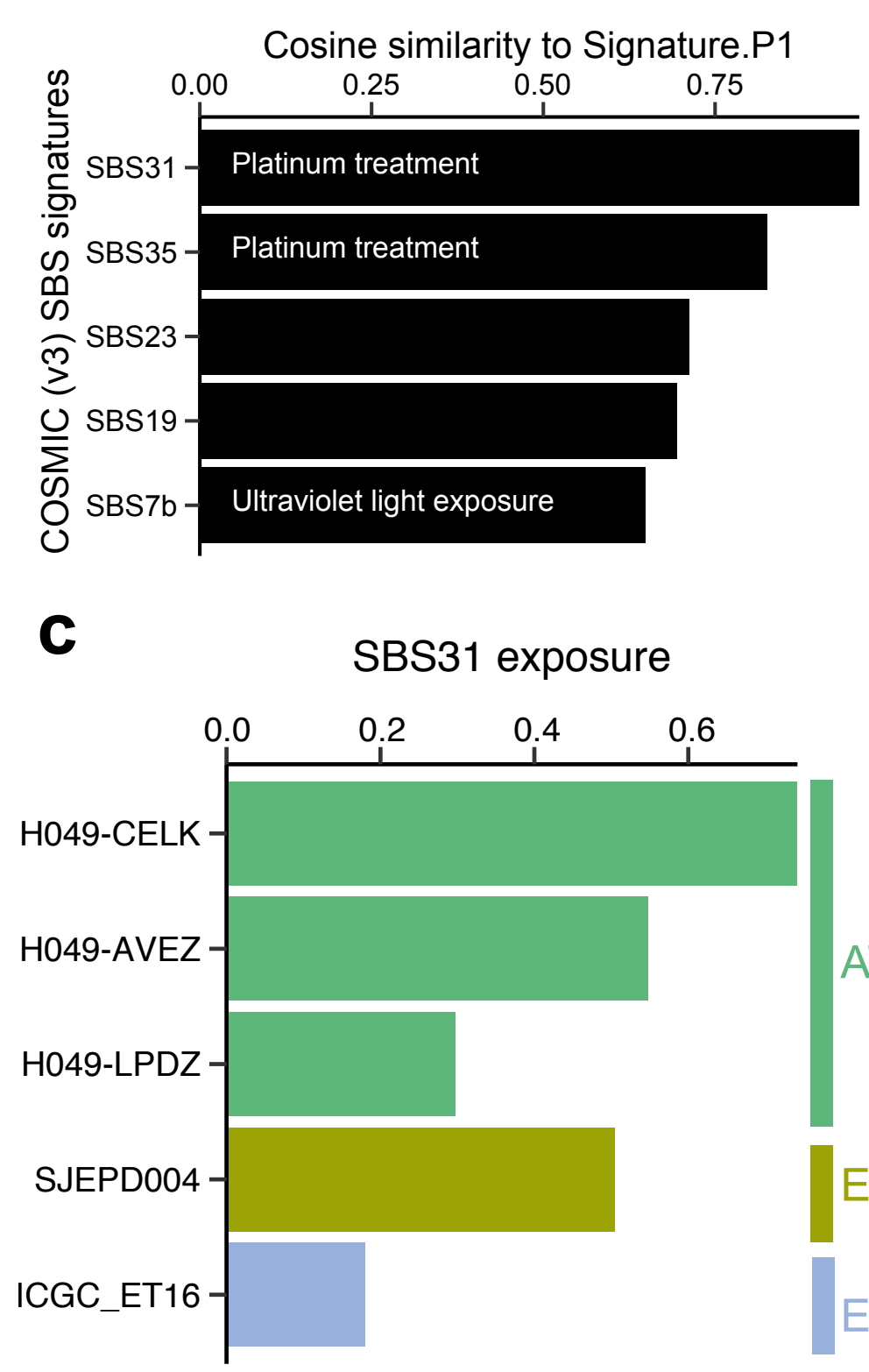

b

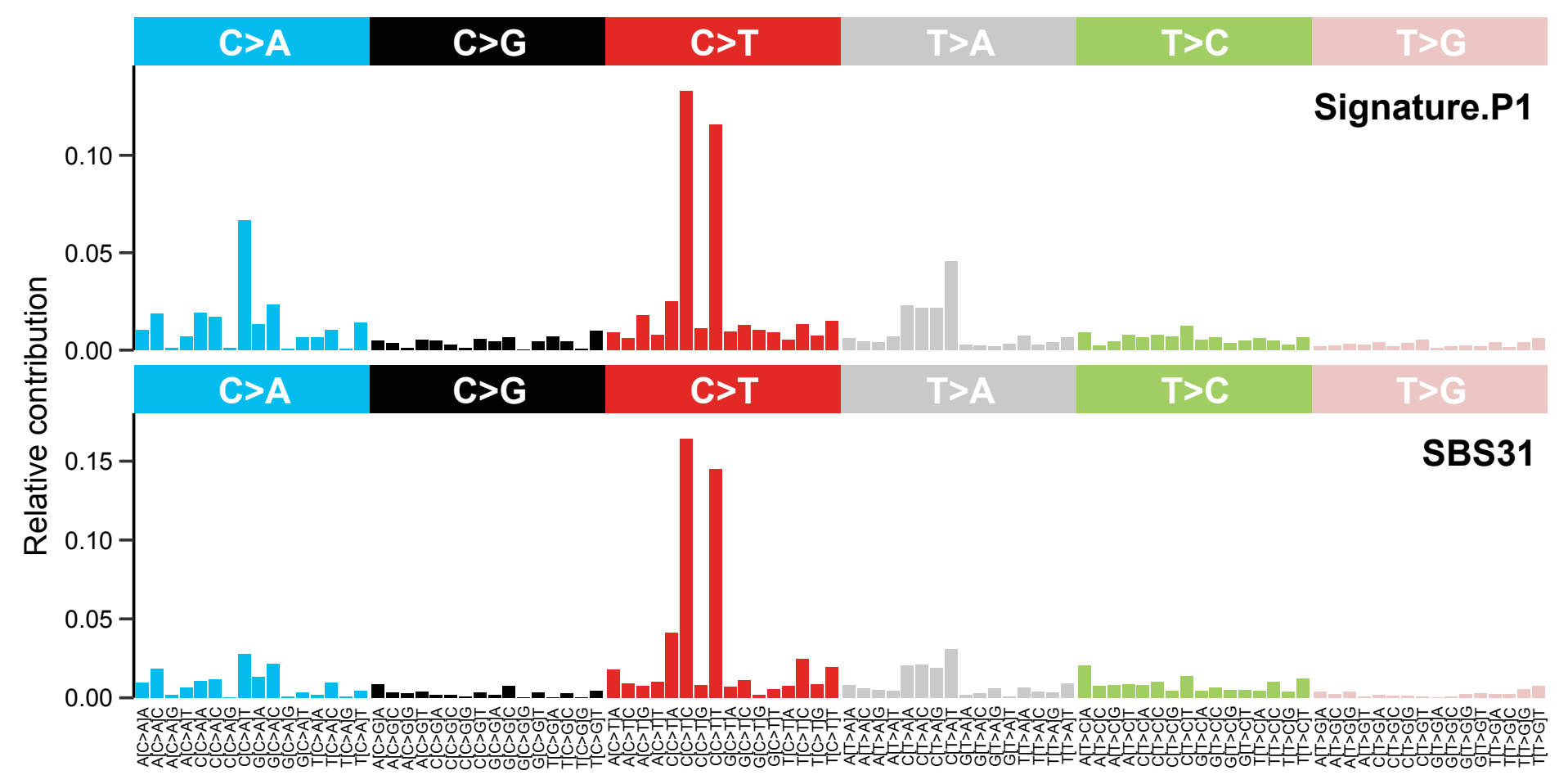

ATRT

Ependymoma (IT) 


\section{Supp Fig. 2:}

a
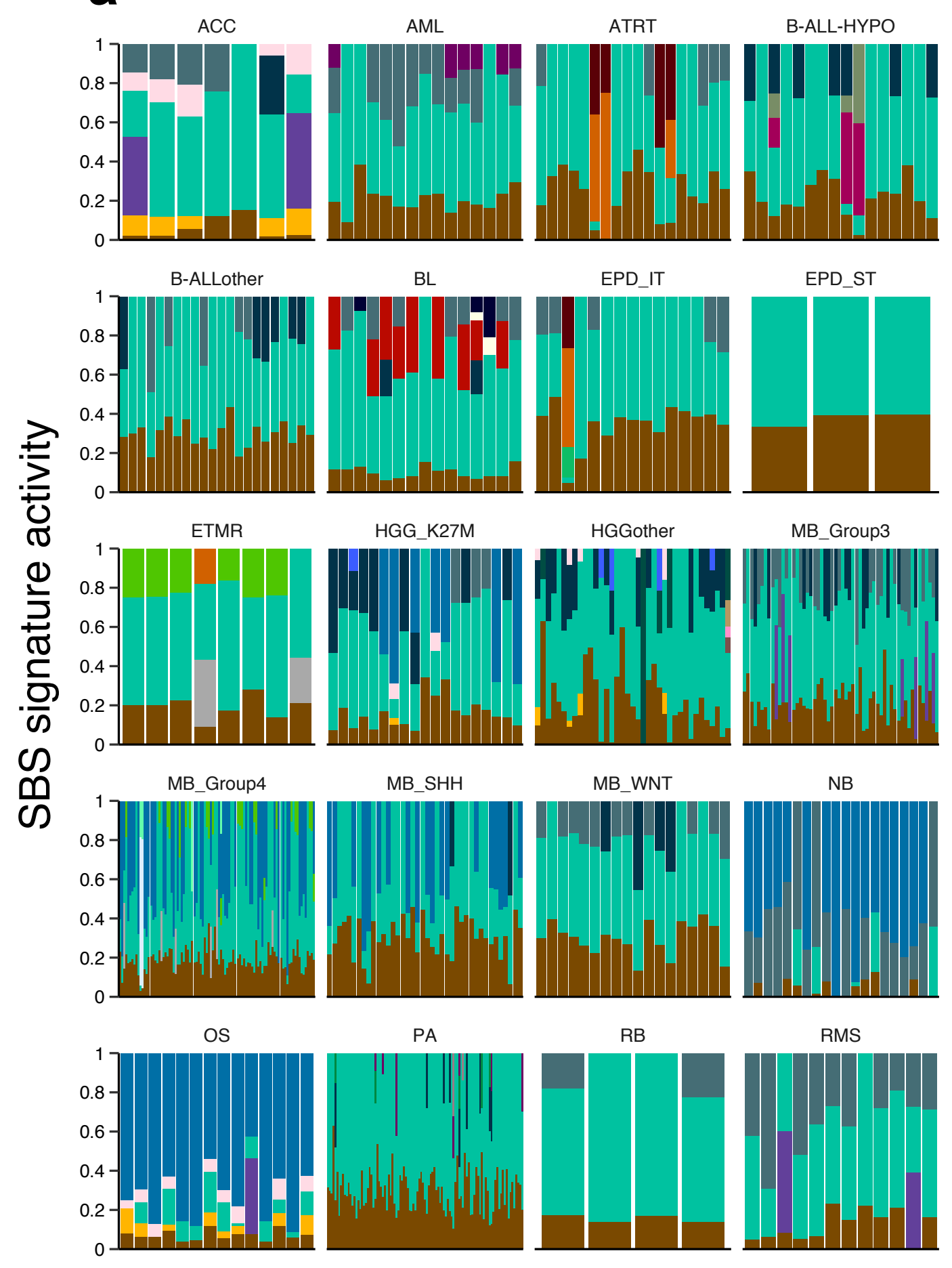

b
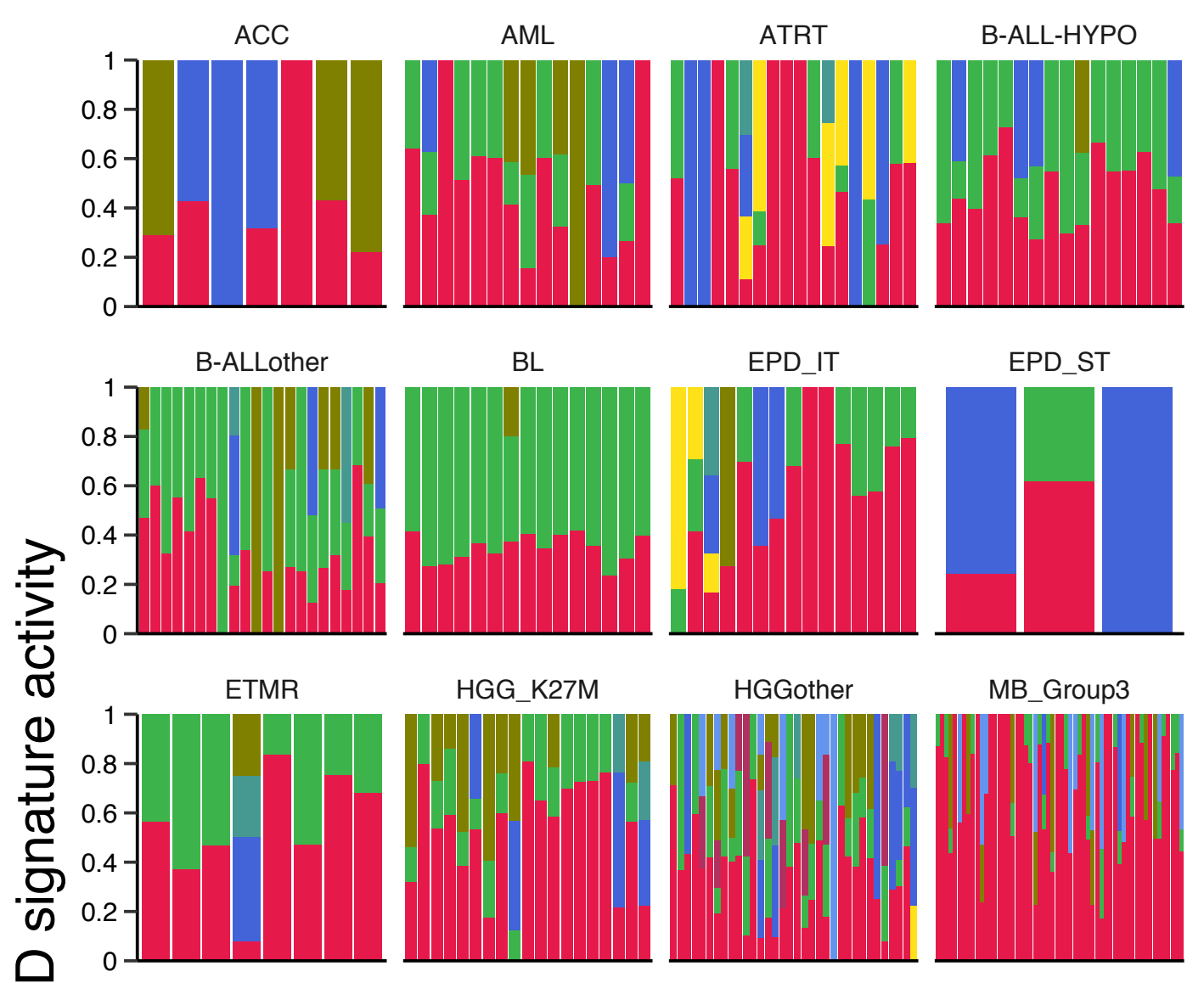

드

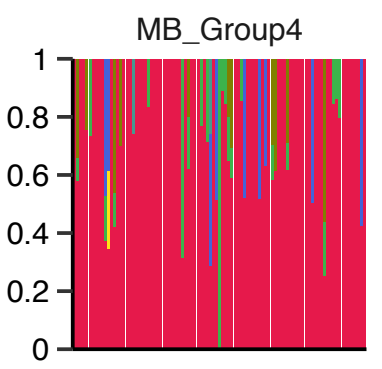

MB_SHH

MB_WNT

NB

SBS15 SBS1

Group4MB-SBS96D
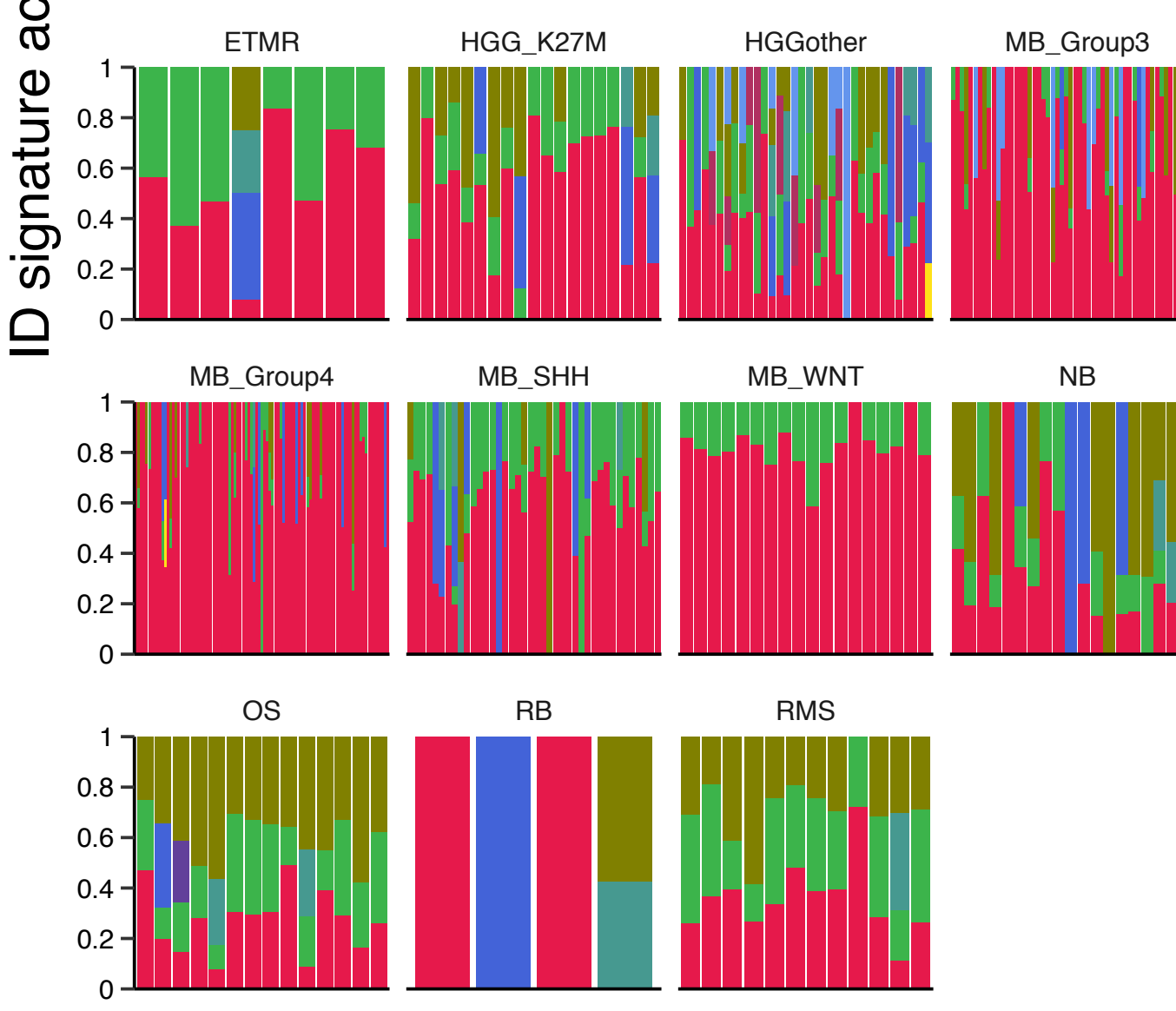


\section{Supp Fig. 5:}

a

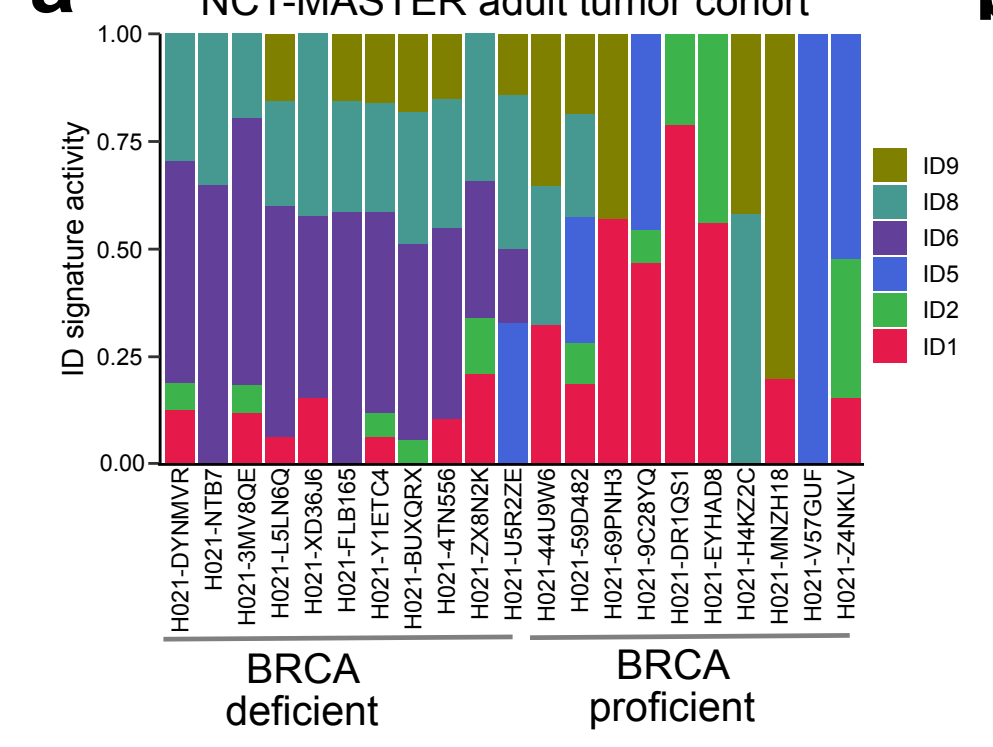

b

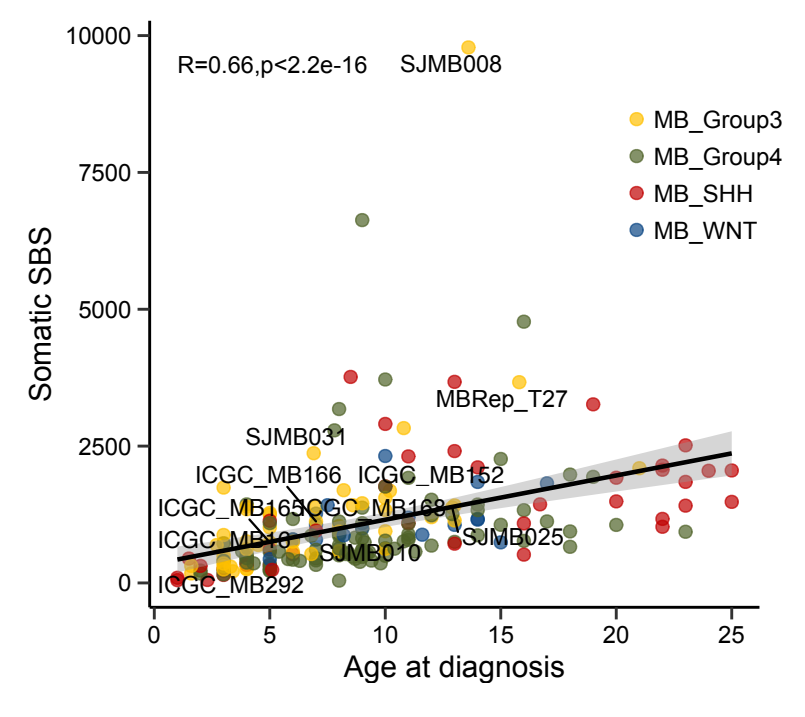

C

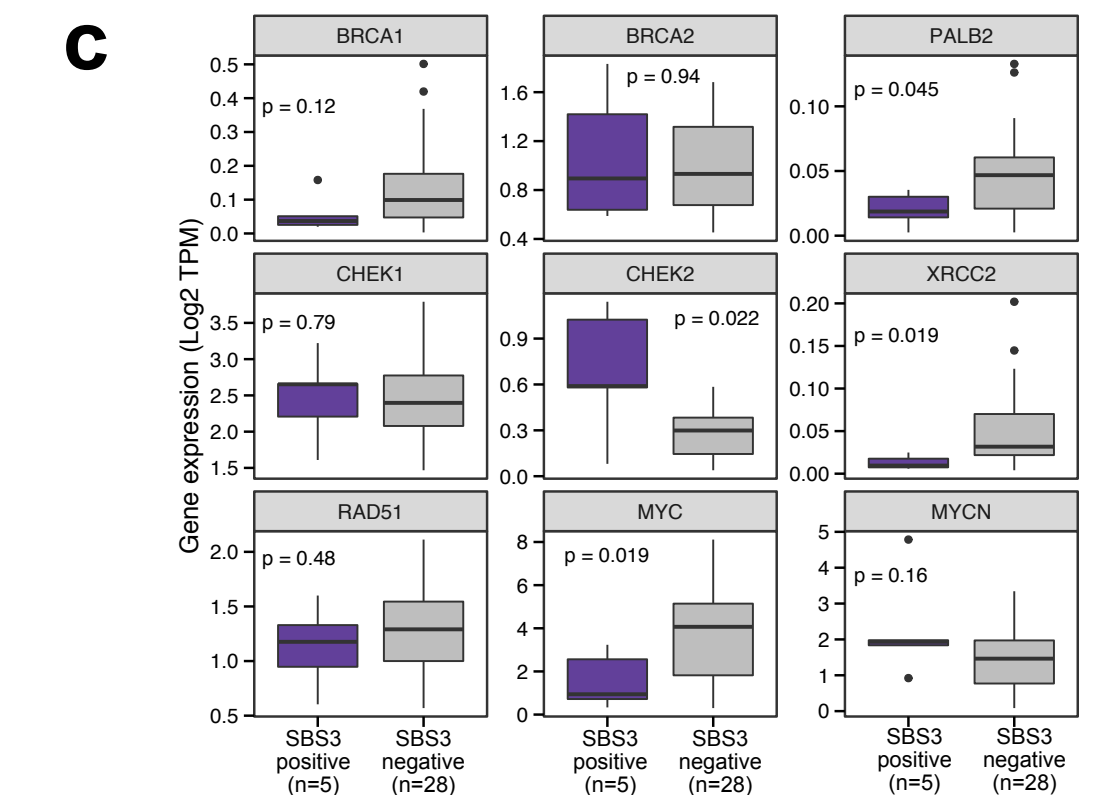

d

\begin{tabular}{lllll} 
& \multicolumn{3}{c}{ Expression in INFORM tumors (TPM) } \\
& Cancer type & BRCA1 & BRCA2 & PALB2 \\
\hline INF_R_1076 & MB_SHH & 20.41 & 2.38 & 22.44 \\
\hline INF_R_025 & HGG & 9.53 & 2.03 & 10.74 \\
\hline INF_R_742 & MB_SHH & 22.06 & 4.33 & 19.54 \\
\hline INF_R_574 & DIPG & 13.68 & 4.38 & 13.77 \\
\hline INF_R_1007 & Alveolar RMS & 19.41 & 10.03 & 19.71 \\
\hline
\end{tabular}


